\title{
Classes of Split-Plot Response Surface Designs for Equivalent Estimation
}

\author{
Peter A. Parker ${ }^{1, *, \dagger}$, Scott M. Kowalski ${ }^{2}$, and G. Geoffrey Vining ${ }^{3}$ \\ ${ }^{1}$ National Aeronautics and Space Administration, Hampton, VA 23681, USA. \\ ${ }^{2}$ Minitab Inc., State College, PA 16801, USA. \\ ${ }^{3}$ Virginia Polytechnic Institute and State University, Blacksburg, VA 24061-0439, USA.
}

\section{Summary}

When planning an experimental investigation, we are frequently faced with factors that are difficult or time consuming to manipulate, thereby making complete randomization impractical. A split-plot structure differentiates between the experimental units associated with these hard-to-change factors and others that are relatively easy-to-change and provides an efficient strategy that integrates the restrictions imposed by the experimental apparatus. Several industrial and scientific examples are presented to illustrate design considerations encountered in the restricted randomization context. In this paper, we propose classes of split-plot response designs that provide an intuitive and natural extension from the completely randomized context. For these designs, the ordinary least squares estimates of the model are equivalent to the generalized least squares estimates. This property provides best linear unbiased estimators and simplifies model estimation. The design conditions that allow for equivalent estimation are presented enabling design construction strategies to transform completely randomized Box-Behnken, equiradial, and small composite designs into a split-plot structure.

KEY WORDS: Restricted Randomization; Split-Plot Designs; Equivalent Estimation; BoxBehnken Design; Equiradial Design; Small Composite Design.

\footnotetext{
* Correspondence to: Peter A. Parker, NASA Langley Research Center, Mail Stop 238, Hampton, Virginia 23681, U.S.A.

${ }^{\dagger}$ E-mail: peter.a.parker@larc.nasa.gov
} 


\section{Introduction}

Response surface methodology (RSM) is widely used to characterize and optimize products and processes. One reason that RSM enjoys success is its recognition of the unique opportunities and restrictions in the industrial context. For example, a sequential strategy is employed where small exploratory experiments are used to guide the design of larger more comprehensive experiments. Motivated by these opportunities, Box and Wilson [1] adapted agricultural methods and developed new experimental strategies tailored to the industrial context. In a similar way, split-plot designs provide an efficient statistical method that recognizes the common occurrence of hard- or costly-to-change factors resulting in a practical restriction on randomization.

In a split-plot design (SPD), we employ two layers of randomization to reduce the number of times that the hard-to-change (HTC) factors are reset. This is a generalization of a factorial experiment in which we have two types of experimental units. First, the whole-plot factors are randomly assigned to the whole-plot experimental units, which with replication generates the whole-plot error variance, $\sigma_{\delta}^{2}$. Then, the subplot factors are randomly assigned

to experimental units within each whole-plot and generates the subplot error variance, $\sigma_{\epsilon}^{2}$. For a more detailed development of industrial split-plot designs see Montgomery [2] and Myers and Montgomery [3].

In traditional response surface designs, all factors are assumed to be equivalent in terms of ease of manipulation. In this paper, we consider experimental situations where this is not the case and explore split-plot designs that accommodate the limitations of the experimental apparatus or process. This is similar to the practice of blocking, designed to reduce the effects of variability associated with nuisance factors. A block consists of a group of experimental runs executed under relatively homogenous conditions. Blocking boundaries, such as days or batches, are naturally determined by the restrictions of the experimental process. A split-plot design is similar in structure to a blocked design if we view the whole-plots as 
blocks. However, in a split-plot design we randomly assign factor combinations to the wholeplots enabling the estimation and testing of whole-plot effects, in contrast to reducing or eliminating them.

Often there exists a natural whole-plot size determined by the experimental apparatus or process, denoted by $n$. For example, if a temperature chamber can accommodate four subplot experimental units, then it would be natural to utilize a design with a whole-plot size of four resulting in $n_{\min }=n_{\max }=n=4$. If the hard-to-change factors are truly hard to change (in contrast to inconvenient to change), then a minimum number of subplot runs, $n_{\min }$, should be executed for each setting of the HTC factors. When we evaluate the size of a split-plot design, we consider the two types of experimental units and their respective costs. For example, if the subplots are significantly less expensive than the whole-plots, then the overall cost of the experiment is dominated by the number of whole-plots rather than the number of subplot runs. Finally, if $n_{\min }=1$, then the completely randomized context is more appropriate.

\section{Illustrations of Restricted Randomization}

The following examples illustrate the diversity of industrial applications and motivate the number of factors encountered in practice. In Vining, Kowalski, and Montgomery [4] a sintering process for the nickel battery plates used in a nickel-hydrogen battery is discussed. In this application, the furnace temperature and belt speed are HTC factors. Once these factors are set, two composition factors, the density of nickel powder and the amount of binder, are relatively easy-to-change (ETC). Once the furnace is stabilized at a specified temperature and belt speed, many samples with different composition levels are processed. In addition, Vining, Kowalski, and Montgomery [4] analyze an experiment to determine the effects of two HTC and two ETC factors on the strength of ceramic pipe.

Letsinger, Myers, and Lentner [5] discuss a chemical processing experiment to study the effect of five factors on a measure of performance. In this experiment temperature and 
pressure are HTC, while the other three factors are relatively ETC. A central composite design in five factors was executed in a split-plot structure. In addition, some factors could not be set to the specified design points, although their levels were measured and utilized in the analysis. This example illustrates a need for a design with performance that is robust to set-point deviations.

Simpson, Kowalski, and Landman [6] consider a wind tunnel experiment involving a race car. They study the impact of four factors (front ride height, rear ride height, yaw angle, and grille configuration) on the aerodynamic performance. In this study, the front and rear ride height factors are hard-to-change because they required considerable time to manipulate. In contrast, the yaw angle and grille configuration were easy to manipulate. A second-order regression model was developed from the wind tunnel tests that could be used to optimize the vehicle performance for a given set of track conditions.

Micol [7] discusses an experiment to characterize the yawing moment (response) produced by the vertical tail configuration of a fighter aircraft as a function of five aerodynamic variables. The factors investigated in a wind tunnel experiment are Mach number, Reynolds number, angle of attack, angle of sideslip, and rudder deflection. Changing the rudder deflection is a manual process requiring a costly and time consuming personnel access into the pressurized test section, on the order of 1.5 hours, thereby making it a HTC factor. In addition to the time, the facility power requirements to re-stabilize the tunnel conditions after each manipulation of this factor represent a significant cost. Alternatively, the other four factors can be set remotely within 15 minutes, and therefore are relatively ETC. In this example, we see that one setting of the HTC factor is equivalent to 6 settings of the other four factors from a time perspective. An experimental strategy that minimizes the number of settings of the HTC factor is required. 


\section{Second-Order Split-Plot Designs}

Letsinger, Myers, and Lentner [5] emphasized the need for second-order split-plot designs and focused on analysis issues; recommending the use of restricted maximum likelihood for model estimation. Draper and John [8] considered modification of the central composite design (CCD) (Box and Wilson [1]) to be executed in a split-plot mode to achieve nearrotatability resulting in a CUBE or STAR structure. Trinca and Gilmour [9] studied multiple layers of split-plotting, called multistratum designs, where the two-level split-plot design is a special case of this general class. They employed a computer-intensive search algorithm to construct designs that satisfied a minimax criterion of the parameter estimate variance while maintaining near-orthogonality between strata. Goos and Vandebroek $[10,11]$ proposed the use of a point exchange algorithm to construct $\mathcal{D}$-optimal split-plot designs, which minimize the volume of the joint confidence region of regression coefficients. Goos [12] provides a thorough development of the $\mathcal{D}$-optimal split-plot design approach including implementation strategies.

Vining, Kowalski, and Montgomery [4], hereafter referred to as VKM, proposed a class of second-order SPD's where ordinary least squares (OLS) is equivalent to generalized least squares (GLS) for model estimation. In addition, VKM proposed augmentation of these designs to provide estimates of pure-error at both the whole-plot and subplot levels. These equivalent estimation designs offer numerous benefits. For example, they can be constructed such that the equivalence property is independent of the model and variance components. Moreover, the parameter estimates are BLUE (best linear unbiased estimators), independent of the variance components, and robust to the assumption of normality. With equivalent estimation designs, model estimation is simplified using OLS, which is available in all statistical software packages.

Parker, Kowalski, and Vining [13] provided a generalized derivation of the equivalence conditions that leads to the development of systematic construction strategies and the ability 
to numerically verify that an arbitrary design achieves the equivalence property. They illustrated the construction of split-plot versions of the central composite design. In addition, they demonstrated the utility of numerical verification with a class of equivalent estimation $\mathcal{D}$-optimal designs.

In this paper, we review the conditions under which ordinary least squares is an appropriate method of estimation for a split-plot design. From these conditions, we illustrate two systematic design construction strategies for a split-plot Box-Behnken (Box and Behnken [14]) design and discuss cases where a crossed construction is useful. In addition, the ability to numerically verify the equivalence property is demonstrated for small composite designs.

We focus on balanced designs where the whole-plots are of equal size. The experimental context determines whether an unbalanced design, with different whole-plot sizes, is a viable option. For example, if the subplot experimental units are not significantly less expensive than the whole-plots, then the reduction in the total number of subplot runs is desirable. However, we believe that in most cases balanced designs are more attractive to the practitioner, straightforward to execute, and may help to ensure homogeneity among whole-plot experimental units.

\section{Equivalence Conditions}

We define the general form of the model as

$$
\mathbf{y}=\mathbf{X} \boldsymbol{\beta}+\boldsymbol{\delta}+\boldsymbol{\epsilon}
$$

where $\mathbf{y}$ is the $N \times 1$ vector of responses, $\mathbf{X}$ is the $N \times p$ model matrix with rank of $p, \boldsymbol{\beta}$ is the $p \times 1$ vector of coefficients, $\boldsymbol{\delta}$ is the $N \times 1$ vector of random whole-plot errors, $\boldsymbol{\epsilon}$ is the $N \times 1$ vector of random subplot errors, $N$ is the total number of subplot runs, and $p$ is the number of terms in the model including the intercept. We assume that $\boldsymbol{\delta}+\boldsymbol{\epsilon}$ has a mean of $\mathbf{0}$ and variance-covariance matrix $\boldsymbol{\Sigma}=\sigma_{\epsilon}^{2} \mathbf{I}+\sigma_{\delta}^{2} \mathbf{J}_{b}$, where $\sigma_{\epsilon}^{2}$ is the subplot error variance, and $\sigma_{\delta}^{2}$ is the whole-plot error variance. In the balanced case, $\mathbf{J}_{b}=\mathbf{I}_{m} \otimes \mathbf{J}_{n}$, where $m$ is the 
number of whole-plots, $n$ is the number of subplot runs within each whole-plot, and $\mathbf{J}_{n}$ is a $n \times n$ matrix of ones. The matrix $\mathbf{J}_{b}$ has the form of

$$
\mathbf{J}_{b}=\left[\begin{array}{cccc}
\mathbf{1}_{n} \mathbf{1}_{n}^{\prime} & 0 & \cdots & 0 \\
0 & \mathbf{1}_{n} \mathbf{1}_{n}^{\prime} & \cdots & 0 \\
\vdots & \vdots & \ddots & \vdots \\
0 & 0 & \cdots & \mathbf{1}_{n} \mathbf{1}_{n}^{\prime}
\end{array}\right]
$$

Examining the variance-covariance structure, reveals that observations in different wholeplots are independent, while observations within a whole-plot are correlated.

Parker, Kowalski, and Vining [13] provide a detailed derivation of the equivalence condition, which we now summarize. We assume that there is one level of split-plotting and that the design supports the model selected. The necessary and sufficient condition for equivalence from McElroy [15] (see also Graybill ([16], p. 209) is

$$
\mathrm{XF}=\Sigma \mathrm{X}
$$

where $\mathbf{F}$ is a $p \times p$ non-singular matrix. We define $\mathbf{F}$ to be

$$
\mathbf{F}=\sigma_{\epsilon}^{2} \mathbf{I}+\sigma_{\delta}^{2} \mathbf{K}
$$

where,

$$
\mathbf{K}=\left(\mathbf{X}^{\prime} \mathbf{X}\right)^{-1} \mathbf{X}^{\prime} \mathbf{J}_{b} \mathbf{X}
$$

It can be shown that the condition for equivalence can be expressed as

$$
\mathbf{X K}=\mathbf{J}_{b} \mathbf{X}
$$

The right-hand side of this equation sums the columns of the model matrix within each whole-plot. These column totals, or unscaled whole-plot means, must equal the model matrix multiplied by $\mathbf{K}$ to satisfy the condition of equivalence. Note that the equivalence condition is independent of the variance components, $\sigma_{\epsilon}^{2}$ and $\sigma_{\delta}^{2}$. 
For a complete second-order model, let $\mathbf{X}$ be partitioned as

$$
\mathbf{X}=\left[\begin{array}{ccccc}
\mathbf{1} & \mathbf{W}_{\mathrm{D}_{1}} & \mathbf{W}_{\mathrm{Q}_{1}} & \mathbf{S}_{\mathrm{D}_{1}} & \mathbf{S}_{\mathrm{Q}_{1}} \\
\mathbf{1} & \mathbf{W}_{\mathrm{D}_{2}} & \mathbf{W}_{\mathrm{Q}_{2}} & \mathbf{S}_{\mathrm{D}_{2}} & \mathbf{S}_{\mathrm{Q}_{2}} \\
\vdots & \vdots & \vdots & \vdots & \vdots \\
\mathbf{1} & \mathbf{W}_{\mathrm{D}_{m}} & \mathbf{W}_{\mathrm{Q}_{m}} & \mathbf{S}_{\mathrm{D}_{m}} & \mathbf{S}_{\mathrm{Q}_{m}}
\end{array}\right]
$$

where the first column corresponds to the intercept, $\mathbf{W}_{\mathrm{D}_{i}}$ denotes the whole-plot main effects and two-factor interactions, $\mathbf{W}_{\mathrm{Q}_{i}}$ contains the whole-plot pure quadratics, $\mathbf{S}_{\mathrm{D}_{i}}$ includes the subplot main effects, subplot by subplot two-factor interactions, and the whole-plot by subplot interactions, and $\mathbf{S}_{\mathrm{Q}_{i}}$ denotes the subplot pure quadratic terms of the $i^{\text {th }}$ whole-plot.

Consider one choice for $\mathbf{K}_{(p \times p)}$ of the form

$$
\mathbf{K}=\left[\begin{array}{ccccc}
n & \mathbf{0}^{\prime} & \mathbf{0}^{\prime} & \mathbf{0}^{\prime} & \mathbf{m}_{\mathbf{0}}^{\prime} \\
\mathbf{0} & n \mathbf{I}_{\mathrm{D}} & \mathbf{0} & \mathbf{0} & \mathbf{0} \\
\mathbf{0} & \mathbf{0} & n \mathbf{I}_{\mathrm{Q}} & \mathbf{0} & \mathbf{M}_{\mathrm{W}} \\
\mathbf{0} & \mathbf{0} & \mathbf{0} & \mathbf{0} & \mathbf{0} \\
\mathbf{0} & \mathbf{0} & \mathbf{0} & \mathbf{0} & \mathbf{V}_{\mathrm{S}}
\end{array}\right]
$$

where $n$ is the number of subplot runs per whole-plot, $\mathbf{m}_{\mathbf{0}}$ is a vector of length $k, \mathbf{I}_{\mathrm{D}}$ and $\mathbf{I}_{\mathrm{Q}}$ are $\left(w+\frac{w(w-1)}{2}\right) \times\left(w+\frac{w(w-1)}{2}\right)$ and $w \times w$ identity matrices, respectively, $\mathbf{M}_{\mathrm{W}}$ is a $w \times k$ matrix, $\mathbf{V}_{\mathrm{S}}$ is a $k \times k$ matrix, where $w$ is the number of whole-plot factors, and $k$ is the number of subplot factors.

By performing the multiplication in Equation (4), we see that, the conditions to achieve equivalence are isolated to the relationships between the within whole-plot column sums of the intercept and the whole-plot and subplot pure quadratic terms.

If the design possesses the equivalence property, then $\hat{\boldsymbol{\beta}}_{O L S}=\hat{\boldsymbol{\beta}}_{G L S}$ and ordinary least squares (OLS) is an appropriate method of estimation. The OLS estimate of the model coefficients is $\hat{\boldsymbol{\beta}}_{O L S}=\left(\mathbf{X}^{\prime} \mathbf{X}\right)^{-1} \mathbf{X}^{\prime} \mathbf{y}$. This expression highlights that the parameter estimates from an equivalent estimation split-plot design are independent of the variance components. By equivalence to GLS, the variance-covariance matrix for the OLS estimate is $\left(\mathbf{X}^{\prime} \mathbf{\Sigma}^{-1} \mathbf{X}\right)^{-1}$. 


\section{Systematic Design Construction Techniques}

Defining the elements of $\mathbf{K}$ based on the parent completely randomized design (CRD) and the construction technique leads to systematic approaches for building equivalent estimation split-plot designs. In this section, we illustrate the construction method proposed by Vining, Kowalski, and Montgomery [4], subsequently referred to as the VKM method, the minimum whole-plot (MWP) method proposed by Parker, Kowalski, and Vining [13], and the crossed construction method.

The notation $\mathrm{D}(w, k)$ is used to denote a design with $w$ whole-plot factors and $k$ subplot factors. Designs in the range of $\mathrm{D}(w, k)$ with $w \in\{1,2,3\}$ and $k \in\{1,2,3,4\}$ are considered.

\subsection{VKM Method Box-Behnken}

For the Box-Behnken (BBD), we restrict our discussion to $w+k=f \leq 5$, since the BBD for $f>5$ is based on a partially balanced incomplete block design and does not produce a consistent form of $\mathbf{K}$. However, with a suitable assignment of the whole-plot factors to the columns of the CRD version of the BBD, the construction methods discussed can be applied directly to produce equivalent estimation designs.

To construct a design of this type, we begin with a completely randomized BBD in $f$ factors. The design is rearranged such that the HTC factors remain constant within each whole-plot (WP) and the subplot designs are orthogonal for a first-order plus interaction model. The center points, consisting of all factors at their zero level in coded units, are placed in a separate whole-plot. To maintain balance, subplot runs are replicated as required within each whole-plot. Finally, the base design can be augmented by replicating the whole-plot containing all center points to provide a pure-error estimate of the whole-plot variance.

To minimize the number of whole-plots, we use a single whole-plot with the HTC factors simultaneously set at it their center level in combination with the pairs of subplot factors 
forming $2^{2}$ factorial combinations. The size of this whole-plot, for $f \leq 5$, is

$$
n_{\max }=2^{2}\left(\begin{array}{c}
k \\
2
\end{array}\right)=2 k(k-1)
$$

In the other whole-plots, with the HTC factors at \pm 1 , replicates of the base subplot design may be required to balance the design. Using this strategy, the whole-plot size can become quite large and may not be practical in certain applications. In these cases additional wholeplots of smaller size could be used. Nevertheless, we assume that these minimum whole-plot designs are permissible.

We define $\mathbf{K}$ for this structure by setting $\mathbf{m}_{\mathbf{0}}=\mathbf{0}$ and $\mathbf{M}_{\mathrm{W}}=\mathbf{0}$. By performing the multiplication in Equation (4) the conditions that must be satisfied are

$$
\begin{aligned}
n \mathbf{W}_{\mathrm{D}_{i}} & =11^{\prime} \mathbf{W}_{\mathrm{D}_{i}} \\
n \mathbf{W}_{\mathrm{Q}_{i}} & =11^{\prime} \mathbf{W}_{\mathrm{Q}_{i}}
\end{aligned}
$$

and

$$
\mathbf{1 1}^{\prime} \mathrm{S}_{\mathrm{Q}_{i}}=\mathbf{S}_{\mathrm{Q}_{i}} \mathbf{V}_{\mathrm{S}} \quad \forall i
$$

For the first set of conditions, since whole-plot factors are held constant within each wholeplot, the column sums of the whole-plot model matrix equals $n$ times the level of the wholeplot model term. These whole-plot model conditions are satisfied for any design by the split-plot structure.

To define $\mathbf{V}_{\mathrm{S}}$, we consider the column sums of the pure quadratic terms in each whole-plot and the condition specified in Equation (7) and find

$$
\mathbf{V}_{\mathrm{S}}=2(k-1) \mathbf{1}_{k} \mathbf{1}_{k}^{\prime}
$$

As an example, consider a design for $\mathrm{D}(1,2)$. Following the VKM construction method we begin with a completely randomized BBD in 3 factors, rearrange the design, and add subplot center replicates as required to balanced. The resulting design has $m=4$ whole-plots of size 
$n=4$ and is shown in Table A.1. To specify the $\mathbf{K}$ matrix, we find $n=4$ and $\mathbf{V}_{\mathrm{S}}=2 \mathbf{1}_{2} \mathbf{1}_{2}^{\prime}$, which satisfies the conditions.

The VKM construction can be applied when $f>5$, however the $\mathbf{K}$ matrix takes on a slightly different form due to the partially balanced nature of the parent design. For the $\mathrm{D}(1,3)$ and $\mathrm{D}(2,3)$ cases, a VKM construction BBD has not been identified. For the $\mathrm{D}(1,3)$ case, a pseudo-crossed approach can be employed where a BBD in the subplot factors is crossed with the three levels of the whole-plot factor. The resulting design contains three wholeplots with identical subplot designs and one whole-plot containing all centers, see Table A.2. This strategy can also be applied for the $\mathrm{D}(2,3)$ case. Note that in the case of $\mathrm{D}(3,3)$ and $\mathrm{D}(3,4)$, the subplot pure quadratics are whole-plot level terms.

The VKM construction method provides a flexible approach to design construction. The equivalency property of the design using the VKM method is independent of the model; it assumes only that the design supports the model. With the VKM method, a subplot design that contains center points, must contain only center points.

\subsection{MWP Method Box-Behnken Designs}

In this section, we propose a class of equivalent estimation designs that require a minimum number of whole-plots. To compute the minimum number of whole-plots required for a BBD, we consider all combinations of the WP factors at their three-levels $(-1,0,+1)$. For $w=1$, $m_{\min }=3$, and for $w>1$ and $f \leq 5$,

$$
m_{\min }=4\left(\begin{array}{c}
w \\
2
\end{array}\right)+2 w+1
$$

For example, the minimum number of whole-plots for the $\mathrm{D}(2,2)$ case requires, $m_{\min }=$ $4(1)+4+1=9$. Note that for $f>5$, the BBD is based on a partially balanced incomplete block design and the number of whole-plots depends on the assignment of the whole-plot factors to the columns of the completely randomized design. The development that follows is based on $f \leq 5$. The construction of designs for $f>5$ is not described; however, a summary 
table of these designs is provided.

To generate an equivalent SPD, we begin with the completely randomized design and proceed in the manner previously discussed by rearranging and ensuring orthogonal subplot designs. However, for the MWP method construction, subplot center runs can be added one-by-one to each whole-plot maintaining balance.

For the MWP class of designs, $\mathbf{K}$ is defined by setting $\mathbf{V}_{\mathrm{S}}=\mathbf{0}$ in Equation (6). Then, by performing the multiplication in Equation (4) we find the condition that must be satisfied, in addition to the conditions involving the whole-plot model matrix, is

$$
\mathbf{1}_{n} \mathbf{1}_{n}^{\prime} \mathbf{S}_{\mathrm{Q}_{i}}=\mathbf{1}_{n} \mathbf{m}_{\mathbf{0}}^{\prime}+\mathbf{W}_{\mathrm{Q}_{i}} \mathbf{M}_{\mathrm{W}} \quad \forall i
$$

Consider the types of whole-plots based on the level and combinations of the whole-plot (WP) and subplot (SP) factors defined by the following three cases;

1. All WP factors simultaneously at their center level in combination with SP factors at their \pm 1 and center level.

2. Two WP factors at their \pm 1 level simultaneously with the SP factors at their center level.

3. A WP factor at \pm 1 in combination with the other WP factors at their center level and one SP factor at its \pm 1 level.

Note that Case (2) is not present for $w=1$.

It can be shown, that the column sum of the subplot pure quadratic terms for Case (1) is $4(k-1)$ and $\mathbf{W}_{\mathrm{Q}_{i}}$ is a matrix of zeroes. Therefore, $\mathbf{m}_{\mathbf{0}}=4(k-1) \mathbf{1}_{k}$. For Case $(2)$, the column sum equals zero and $\mathbf{W}_{\mathrm{Q}_{i}}=\left[\begin{array}{llll}\mathbf{1}_{n} & \mathbf{1}_{n} & \cdots & \mathbf{0}\end{array}\right]_{n \times w}$, where the position of the vectors of ones will change depending on which two WP factors are at their \pm 1 level. For Case (3), the column sum equals 2 and $\mathbf{W}_{\mathrm{Q}_{i}}=\left[\begin{array}{llll}\mathbf{1}_{n} & \mathbf{0} & \cdots & \mathbf{0}\end{array}\right]_{n \times w}$, where the position of the vector of ones will change depending on which WP factor is at its \pm 1 level. We define 
$\mathbf{M}_{\mathrm{W}}$ to be of the form $\phi \mathbf{1}_{w} \mathbf{1}_{k}^{\prime}$. For Case (2), we find

$$
\phi=-\frac{4(k-1)}{2}
$$

For Case (3), we find

$$
\phi=6-4 k .
$$

Therefore, we summarize the conditions as

$$
\phi=\left\{\begin{array}{ll}
6-4 k & \text { if } w=1 \\
-\frac{4(k-1)}{2}=6-4 k & \text { if } w>1, k>1
\end{array} .\right.
$$

As a result of this restriction on $\phi$, MWP method BBD's are not permissible for $\mathrm{D}(2,3)$, $\mathrm{D}(2,4), \mathrm{D}(3,3)$, and $\mathrm{D}(3,4)$. In addition, other special cases include $\mathrm{D}(2,1)$ and $\mathrm{D}(3,1)$, where the MWP BBD is equivalent to the VKM construction BBD.

We note that the relationship to achieve equivalence is not dependent on the whole-plot size, $n$. Therefore, adding subplot center runs to each whole-plot does not change the column sums, and thereby maintains the equivalence property.

Consider a MWP BBD for $\mathrm{D}(1,2)$. We find that $m_{\min }=3, n=5, \mathbf{m}_{\mathbf{0}}=4 \mathbf{1}_{2}$, and $\mathbf{M}_{\mathrm{W}}=-2 \mathbf{1}_{2}^{\prime}$. The design is shown in Table A.3. Comparing this design to the VKM method BBD for $\mathrm{D}(1,2)$, we see that there is one less whole-plot and the the whole-plot size has increased by one subplot run.

Now, consider including another HTC factor to the design requiring a $\mathrm{D}(2,2)$ design. For this design we find, $m_{\min }=9, n=5, \mathbf{m}_{\mathbf{0}}=4 \mathbf{1}_{2}$, and $\mathbf{M}_{\mathrm{W}}=-2 \mathbf{1}_{2} \mathbf{1}_{2}^{\prime}$, which satisfy the equivalence conditions. A D $(2,2)$ design based on a four factor BBD is shown in Table A.4. The MWP method establishes the lower boundary for the number of whole-plots required to conduct a Box-Behnken design in split-plot mode. In addition, its structure allows for subplot centers to be included within each whole-plot, and the subplot centers can be added 
one-by-one to each whole-plot maintaining the equivalence property. (Note that this feature is not available for $\mathrm{D}(2,1)$ and $\mathrm{D}(3,1)$, which are identical to the VKM method designs and therefore do not allow subplot centers to be added one-by-one.) Finally, a wholeplot containing all center points for both the whole-plot and subplot factors violates the equivalence condition and is not permissible.

Table B.1 provides a summary of the design size for the VKM and MWP method construction split-plot BBD's for all combinations of $1 \leq w \leq 3$ and $1 \leq k \leq 4$, where a design has been identified.

\subsection{Crossed Designs}

It is well-known that OLS is an appropriate method of estimation of a first-order plus interaction model for a crossed design with orthogonal subplot designs. Bisgaard [17] discusses these designs as a Cartesian product and Taguchi [18] refers to them as product arrays. From our derivation, we can show that the equivalent estimation property also holds for second-order designs. In the model matrix $\mathbf{X}$, we see that $\mathbf{S}_{\mathrm{Q}_{i}}=\mathbf{S}_{\mathrm{Q}}, \forall i$. Referring to $\mathbf{K}$, let $\mathbf{V}_{\mathrm{S}}=\mathbf{0}$ and $\mathbf{M}_{\mathrm{W}}=\mathbf{0}$ and the equivalence condition becomes $\mathbf{1}_{n} \mathbf{1}_{n}^{\prime} \mathbf{S}_{\mathrm{Q}}=\mathbf{1}_{n} \mathbf{m}_{\mathbf{0}}^{\prime}$. Therefore, $\mathbf{m}_{\mathbf{0}}=\mathbf{1}_{n}^{\prime} \mathbf{S}_{\mathrm{Q}}$. Therefore, using identical subplot designs results in an equivalent estimation second-order design, regardless of the specific design chosen.

In many cases, a crossed construction results in an unnecessarily large design. However, a crossed construction can be an attractive alternative if the parent designs involved have relatively few points. In this section we consider crossing combinations of 3-level designs in one factor and equiradial designs in two factors.

We can achieve the minimum design size equiradial design by using a pentagon augmented with a center point to avoid singularity of the moment matrix; center points are essential to all equiradial designs. However, the pentagon is saturated for a second-order model, and therefore it is not recommended unless dictated by cost. Alternatively, a hexagon augmented with a center point is an attractive choice, requiring two fewer design points than a spherical 
central composite design (CCD) and provides one degree of freedom for lack-of-fit. (Note that the spherical CCD in two factors is an equiradial octagon design.) The hexagonal design features one factor at three levels and the other at five levels, providing a nice compromise in the number of required factor levels compared to the CCD.

First we consider designs at the whole-plot level. For $\mathrm{D}(1, k)$, a 3-level design in the wholeplot factors can be crossed with a suitable design in the subplot factors. For $\mathrm{D}(2, k)$ a saturated pentagon plus center point design requires 6 whole-plots, while the hexagon plus center point requires 7 whole-plots. It is straightforward to see for subplot designs that a 3-level design can be applied for $\mathrm{D}(w, 1)$ and a hexagon plus center point for $\mathrm{D}(w, 2)$.

As an example, consider a design for $\mathrm{D}(2,2)$ based on crossing two hexagonal designs at the whole-plot and subplot level shown in Table A.5. This crossed design features two fewer whole-plots than the MWP BBD shown in Table A.4. However, the subplot size has increased from 5 to 7 . Even with this increase in the number of subplot runs, the reduction in the number of whole-plots may be more attractive if the cost of the experiment is dominated by setting the HTC factors. There are no replicates at the whole-plot or subplot level included in this base design. One approach is to augment the whole-plots with replicated subplot centers. In addition, we might consider replicating whole-plot number 7 (whole-plot factors simultaneously at their center level) to obtain a pure-error estimate of whole-plot error variance.

\section{Numerically Verified Small Composite Designs}

For some classes of completely randomized response surface designs, a consistent analytical form of $\mathbf{K}$ does not exist. However, configuring these designs into a split-plot version can be performed by following a systematic construction method and then verifying if the design possess the equivalence property. Using Equation (3) to directly compute $\mathbf{K}$, we can verify if the condition from Equation (4) is satisfied. 
As an example, consider the small composite designs (SCD) proposed by Hartley [19] constructed to reduce the size of the factorial, or cube, portion of a composite design. Draper [20] and Draper and Lin [21] proposed using a subset of columns from a Plackett-Burman design (PBD) and recommend column choices that provide designs with a repeated run and those with high relative $\mathcal{D}$ values in a completely randomized context.

To illustrate the utility of numerical verification using an SCD, consider a split-plot design for $\mathrm{D}(1,4)$. We begin with a five-factor cuboidal SCD based on a PBD factorial that includes a repeated run, which is deleted, to obtain a saturated design. A center point is added to provide one degree of freedom for lack-of-fit, even though it is not essential in a cuboidal design. Applying the MWP method and replicating subplot centers as required to achieve balance, we obtain the split-plot design shown in Table A.6. We compute $\mathbf{K}_{(21 \times 21)}$ directly for a complete second-order model as

$$
\left[\begin{array}{rrrrrrrrrrrrrrr}
\text { int } & z_{1} & z_{1}^{2} & x_{1} & \cdots & x_{4} & x_{1} x_{2} & \cdots & x_{3} x_{4} & z_{1} x_{1} & \cdots & z_{1} x_{4} & x_{1}^{2} & \cdots & x_{4}^{2} \\
9 & 0 & 0 & 0 & \cdots & 0 & 0 & \cdots & 0 & 0 & \cdots & 0 & 2 & \cdots & 2 \\
0 & 9 & 0 & -0.5 & \cdots & -0.5 & -1.5 & \cdots & -1.5 & 0.5 & \cdots & 0.5 & 0.5 & \cdots & 0.5 \\
0 & 0 & 9 & 0.5 & \cdots & 0.5 & -0.5 & \cdots & -0.5 & -0.5 & \cdots & -0.5 & 3.5 & \cdots & 3.5 \\
0 & \cdots & \cdots & \cdots & \cdots & \cdots & \cdots & \cdots & \cdots & \cdots & \cdots & \cdots & \cdots & \cdots & 0 \\
\vdots & & & & & & & & & & & & & & \vdots \\
0 & \cdots & \cdots & \cdots & \cdots & \cdots & \cdots & \cdots & \cdots & \cdots & \cdots & \cdots & \cdots & \cdots & 0
\end{array}\right]
$$

where the first row indicates the partitioning of the model terms within $\mathbf{K}$. Notice that the first whole-plot in Table A.6 does not contain an orthogonal subplot design resulting in non-zero elements associated with the subplot main effects and two-factor interactions in $\mathbf{K}$. Applying Equation (4) we find that the conditions for equivalence are satisfied. Inspecting, this form of $\mathbf{K}$ and considering the equivalence conditions, we can show that subplot centers can be added one-by-one within the whole-plots and maintain the equivalence property.

An alternative design, shown in Table A.7, was constructed using a complete 12-run PBD factorial portion according to the column selection specified by Draper and Lin [21] that provides the highest relative $\mathcal{D}$ value. This design also has 3 whole-plots of size 9 and features orthogonal subplot main effects. While both of these SCD's reduce the number of unique design points, an MWP central composite design based on a resolution $\mathrm{V}$ fraction 
was proposed by Parker, Kowalski, and Vining [13] with 3 whole-plots of size 9, without replication (see Table A.8). From a cost perspective, all of three of these competing designs are equivalent, however, their statistical properties are quite different.

One criterion to compare these three competing designs is their relative $\mathcal{D}$-efficiency, which is a measure of their estimation efficiency. Since the designs have the same number of subplot runs and the same allocation among three whole-plots, we do not need to apply scaling factor. The relative $\mathcal{D}$-efficiency of the minimal point design in Table A.6 is $49.8 \%$ and the complete Plackett-Burman factorial design in Table A.7 is $55.0 \%$ compared to the classical CCD in Table A.8. Even if we increase the subplot size to 10, allowing for subplot level replication in the $\mathrm{CCD}$, these large improvements in efficiency are maintained.

Clearly, the CCD is a better choice from an estimation perspective. In addition, the projection properties of the CCD are more attractive due to the partial aliasing in the PBD. Alternatively, the smaller factorial portion of the SCD provides the ability to estimate subplot pure-error with fewer subplot runs than the CCD. As in most practical situations, compromises among competing criteria in the design selection process must be considered.

\section{Concluding Remarks}

We have emphasized the need to distinguish between HTC and ETC factors and the relative cost of their respective experimental units. Integrating apparatus-based restrictions into the experimental strategy provides an efficient approach to conduct response surface investigations in the presence of restricted randomization.

Equivalent estimation split-plot designs provide simplified and robust estimation that is independent of the variance components. Simplified because industrial practitioners can perform model estimation using OLS software, in contrast to more sophisticated analysis software normally required for split-plot designs. Robust in the sense that the design performance can be independent of the model, and the parameter estimates are best linear unbiased estima- 
tors. Finally, design augmentation providing replication at both the whole-plot and subplot level to enable pure-error estimates of the variance components is straightforward.

Two systematic construction methods to transform a Box-Behnken design into a non-crossed split-plot structure have been presented. Cases where a crossed construction is useful have been highlighted with three-level and equiradial designs. In addition, a numerical verification strategy has been illustrated with small composite designs. A comprehensive catalog containing equivalent estimation split-plot versions of many classical response surface designs is available at the author's website. The construction methods coupled with the pre-built catalog of designs makes equivalent estimation designs available to practitioners.

\section{References}

[1] Box GEP, Wilson KB. On the Experimental Attainment of Optimum Conditions. Journal of the Royal Statistical Society Ser.B 1951; 13: 195-241.

[2] Montgomery DC. Design and Analysis of Experiments (6th edn.). Wiley: New York, 2004.

[3] Myers RH, Montgomery DC, Response Surface Methodology (2nd edn.). Wiley: New York 2002.

[4] Vining GG, Kowalski SM, Montgomery DC. Response Surface Designs Within a SplitPlot Structure. Journal of Quality Technology 2005; 37: 115-129.

[5] Letsinger JD, Myers RH, Lentner M. Response Surface Methods for Bi-Randomization Structures. Journal of Quality Technology 1996; 28: 381-397.

[6] Simpson JR, Kowalski SM, Landman D. Experimentation with Randomization Restrictions: Targeting Practical Implementation. Quality and Reliability Engineering International 2004; 20: 481-495.

[7] Micol JR. Langley Research Center's Unitary Plan Wind Tunnel: Testing Capabilities and Recent Moderization Activities. American Institute of Aeronautics and Astronautics 39th Aerospace Sciences Meeting AIAA-2001-0456, Reno, Nevada 2001. 
[8] Draper NR, John JA. Response Surface Designs where Levels of Some Factors Are Difficult to Change. Australian and New Zealand Journal of Statistics 1998; 40: 487495.

[9] Trinca LA, Gilmour, SG. Multistratum Response Surface Designs. Technometrics 2001; 43: 25-33.

[10] Goos P, Vandebroek M. Optimal Split-Plot Designs. Journal of Quality Technology 2001; 33: $436-450$.

[11] Goos P, Vandebroek M. D-optimal Split-Plot Designs with Given Numbers and Sizes of Whole Plots. Technometrics 2003; 45: 235-245.

[12] Goos P. The Optimal Design of Blocked and Split-Plot Experiments. Springer-Verlag: New York, 2002.

[13] Parker PA, Kowalski SM, Vining GG. Construction of Balanced Equivalent Estimation Second-Order Split-Plot Designs. submitted to Technometrics 2005.

[14] Box GEP, Behnken DW. Some New Three-Level Designs for the Study of Quantitative Variables. Technometrics 1960; 2: 455-475.

[15] McElroy FW. A Necessary and Sufficient Condition that Ordinary Least Squares Estimators Be Best Linear Unbiased. Journal of the American Statistical Association 1967; 62: $1302-1304$.

[16] Graybill, FA. Theory and Application of the Linear Model. Duxbury: North Scituate, MA, 1976.

[17] Bisgaard S. The Design and Analysis of $2^{k-p} \times 2^{q-r}$ Split Plot Experiments Journal of Quality Technology 2000; 32: 39-56.

[18] Taguchi G. System of Experimental Design Vol. 1. Kraus International: White Plains, NY, 1987.

[19] Hartley HO. Small Composite Design for Quadratic Response Surfaces. Biometrika 1959; 15: 611-624.

[20] Draper NR. Small Composite Designs. Technometrics 1985; 27: 173-180.

[21] Draper NR, Lin DKJ. Small Response Surface Designs Technometrics 1990; 32: 187194. 


\section{A Equivalent Estimation Split-Plot Designs}

Table A.1: VKM Method Box-Behnken Design for D $(1,2)$.

$\left(z_{1}\right.$ is a HTC factor, $x_{1}$ and $x_{2}$ are ETC factors)

\begin{tabular}{crrr} 
Whole-Plot & $z_{1}$ & $x_{1}$ & $x_{2}$ \\
\hline 1 & -1 & -1 & 0 \\
& -1 & 1 & 0 \\
& -1 & 0 & -1 \\
& -1 & 0 & 1 \\
\hline 2 & 1 & -1 & 0 \\
& 1 & 1 & 0 \\
& 1 & 0 & -1 \\
& 1 & 0 & 1 \\
\hline 3 & 0 & -1 & -1 \\
& 0 & 1 & -1 \\
& 0 & -1 & 1 \\
& 0 & 1 & 1 \\
\hline 4 & 0 & 0 & 0 \\
& 0 & 0 & 0 \\
& 0 & 0 & 0 \\
& 0 & 0 & 0 \\
\hline
\end{tabular}


Table A.2: VKM Pseudo-Crossed Method Box-Behnken Design for D $(1,3)$. $\left(z_{1}\right.$ is a HTC factor, $x_{1}, x_{2}$, and $x_{3}$ are ETC factors)

\begin{tabular}{|c|c|c|c|c|c|c|c|c|c|}
\hline Whole-Plot & $z_{1}$ & $x_{1}$ & $x_{2}$ & $x_{3}$ & Whole-Plot & $z_{1}$ & $x_{1}$ & $x_{2}$ & $x_{3}$ \\
\hline \multirow[t]{12}{*}{1} & -1 & -1 & -1 & 0 & \multirow[t]{12}{*}{3} & 0 & -1 & -1 & 0 \\
\hline & -1 & 1 & -1 & 0 & & 0 & 1 & -1 & 0 \\
\hline & -1 & -1 & 1 & 0 & & 0 & -1 & 1 & 0 \\
\hline & -1 & 1 & 1 & 0 & & 0 & 1 & 1 & 0 \\
\hline & -1 & -1 & 0 & -1 & & 0 & -1 & 0 & -1 \\
\hline & -1 & 1 & 0 & -1 & & 0 & 1 & 0 & -1 \\
\hline & -1 & -1 & 0 & 1 & & 0 & -1 & 0 & 1 \\
\hline & -1 & 1 & 0 & 1 & & 0 & 1 & 0 & 1 \\
\hline & -1 & 0 & -1 & -1 & & 0 & 0 & -1 & -1 \\
\hline & -1 & 0 & 1 & -1 & & 0 & 0 & 1 & -1 \\
\hline & -1 & 0 & -1 & 1 & & 0 & 0 & -1 & 1 \\
\hline & -1 & 0 & 1 & 1 & & 0 & 0 & 1 & 1 \\
\hline \multirow[t]{12}{*}{2} & 1 & -1 & -1 & 0 & \multirow[t]{12}{*}{4} & 0 & 0 & 0 & 0 \\
\hline & 1 & 1 & -1 & 0 & & 0 & 0 & 0 & 0 \\
\hline & 1 & -1 & 1 & 0 & & 0 & 0 & 0 & 0 \\
\hline & 1 & 1 & 1 & 0 & & 0 & 0 & 0 & 0 \\
\hline & 1 & -1 & 0 & -1 & & 0 & 0 & 0 & 0 \\
\hline & 1 & 1 & 0 & -1 & & 0 & 0 & 0 & 0 \\
\hline & 1 & -1 & 0 & 1 & & 0 & 0 & 0 & 0 \\
\hline & 1 & 1 & 0 & 1 & & 0 & 0 & 0 & 0 \\
\hline & 1 & 0 & -1 & -1 & & 0 & 0 & 0 & 0 \\
\hline & 1 & 0 & 1 & -1 & & 0 & 0 & 0 & 0 \\
\hline & 1 & 0 & -1 & 1 & & 0 & 0 & 0 & 0 \\
\hline & 1 & 0 & 1 & 1 & & 0 & 0 & 0 & 0 \\
\hline
\end{tabular}


Table A.3: MWP Method Box-Behnken Design for D $(1,2)$. ( $z_{1}$ is a HTC factor, $x_{1}$ and $x_{2}$ are ETC factors)

\begin{tabular}{crrr} 
Whole-Plot & $z_{1}$ & $x_{1}$ & $x_{2}$ \\
\hline 1 & -1 & -1 & 0 \\
& -1 & 1 & 0 \\
& -1 & 0 & -1 \\
& -1 & 0 & 1 \\
& -1 & 0 & 0 \\
\hline 2 & 1 & -1 & 0 \\
& 1 & 1 & 0 \\
& 1 & 0 & -1 \\
& 1 & 0 & 1 \\
& 1 & 0 & 0 \\
\hline 3 & 0 & -1 & -1 \\
& 0 & -1 & 1 \\
& 0 & 1 & -1 \\
& 0 & 1 & 1 \\
& 0 & 0 & 0 \\
\hline
\end{tabular}


Table A.4: MWP Method Box-Behnken Design for D $(2,2)$. $\left(z_{1}\right.$ and $z_{2}$ are HTC factors, $x_{1}$ and $x_{2}$ are ETC factors)

\begin{tabular}{|c|c|c|c|c|c|c|c|c|c|}
\hline Whole-Plot & $z_{1}$ & $z_{2}$ & $x_{1}$ & $x_{2}$ & Whole-Plot & $z_{1}$ & $z_{2}$ & $x_{1}$ & $x_{2}$ \\
\hline \multirow[t]{5}{*}{1} & -1 & -1 & 0 & 0 & \multirow[t]{5}{*}{6} & 1 & 0 & 0 & -1 \\
\hline & -1 & -1 & 0 & 0 & & 1 & 0 & 0 & 1 \\
\hline & -1 & -1 & 0 & 0 & & 1 & 0 & -1 & 0 \\
\hline & -1 & -1 & 0 & 0 & & 1 & 0 & 1 & 0 \\
\hline & -1 & -1 & 0 & 0 & & 1 & 0 & 0 & 0 \\
\hline \multirow[t]{5}{*}{2} & 1 & -1 & 0 & 0 & \multirow[t]{5}{*}{7} & 0 & -1 & -1 & 0 \\
\hline & 1 & -1 & 0 & 0 & & 0 & -1 & 1 & 0 \\
\hline & 1 & -1 & 0 & 0 & & 0 & -1 & 0 & -1 \\
\hline & 1 & -1 & 0 & 0 & & 0 & -1 & 0 & 1 \\
\hline & 1 & -1 & 0 & 0 & & 0 & -1 & 0 & 0 \\
\hline \multirow[t]{5}{*}{3} & -1 & 1 & 0 & 0 & \multirow[t]{5}{*}{8} & 0 & 1 & -1 & 0 \\
\hline & -1 & 1 & 0 & 0 & & 0 & 1 & 1 & 0 \\
\hline & -1 & 1 & 0 & 0 & & 0 & 1 & 0 & -1 \\
\hline & -1 & 1 & 0 & 0 & & 0 & 1 & 0 & 1 \\
\hline & -1 & 1 & 0 & 0 & & 0 & 1 & 0 & 0 \\
\hline \multirow[t]{5}{*}{4} & 1 & 1 & 0 & 0 & \multirow[t]{5}{*}{9} & 0 & 0 & -1 & -1 \\
\hline & 1 & 1 & 0 & 0 & & 0 & 0 & -1 & 1 \\
\hline & 1 & 1 & 0 & 0 & & 0 & 0 & 1 & -1 \\
\hline & 1 & 1 & 0 & 0 & & 0 & 0 & 1 & 1 \\
\hline & 1 & 1 & 0 & 0 & & 0 & 0 & 0 & 0 \\
\hline \multirow[t]{5}{*}{5} & -1 & 0 & 0 & -1 & & & & & \\
\hline & -1 & 0 & 0 & 1 & & & & & \\
\hline & -1 & 0 & -1 & 0 & & & & & \\
\hline & -1 & 0 & 1 & 0 & & & & & \\
\hline & -1 & 0 & 0 & 0 & & & & & \\
\hline
\end{tabular}


Table A.5: Crossed Hexagonal Design for $\mathrm{D}(2,2)$.

$\left(z_{1}\right.$ and $z_{2}$ are HTC factors, $x_{1}$ and $x_{2}$ are ETC factors)

\begin{tabular}{|c|c|c|c|c|c|c|c|c|c|}
\hline Whole-Plot & $z_{1}$ & $z_{2}$ & $x_{1}$ & $x_{2}$ & Whole-Plot & $z_{1}$ & $z_{2}$ & $x_{1}$ & $x_{2}$ \\
\hline \multirow[t]{7}{*}{1} & 1 & 0 & 1 & 0 & \multirow[t]{7}{*}{5} & -0.5 & $-\sqrt{0.75}$ & 1 & 0 \\
\hline & 1 & 0 & 0.5 & $\sqrt{0.75}$ & & -0.5 & $-\sqrt{0.75}$ & 0.5 & $\sqrt{0.75}$ \\
\hline & 1 & 0 & -0.5 & $\sqrt{0.75}$ & & -0.5 & $-\sqrt{0.75}$ & -0.5 & $\sqrt{0.75}$ \\
\hline & 1 & 0 & -1 & 0 & & -0.5 & $-\sqrt{0.75}$ & -1 & 0 \\
\hline & 1 & 0 & -0.5 & $-\sqrt{0.75}$ & & -0.5 & $-\sqrt{0.75}$ & -0.5 & $-\sqrt{0.75}$ \\
\hline & 1 & 0 & 0.5 & $-\sqrt{0.75}$ & & -0.5 & $-\sqrt{0.75}$ & 0.5 & $-\sqrt{0.75}$ \\
\hline & 1 & 0 & 0 & 0 & & -0.5 & $-\sqrt{0.75}$ & 0 & 0 \\
\hline \multirow[t]{7}{*}{2} & 0.5 & $\sqrt{0.75}$ & 1 & 0 & \multirow[t]{7}{*}{6} & 0.5 & $-\sqrt{0.75}$ & 1 & 0 \\
\hline & 0.5 & $\sqrt{0.75}$ & 0.5 & $\sqrt{0.75}$ & & 0.5 & $-\sqrt{0.75}$ & 0.5 & $\sqrt{0.75}$ \\
\hline & 0.5 & $\sqrt{0.75}$ & -0.5 & $\sqrt{0.75}$ & & 0.5 & $-\sqrt{0.75}$ & -0.5 & $\sqrt{0.75}$ \\
\hline & 0.5 & $\sqrt{0.75}$ & -1 & 0 & & 0.5 & $-\sqrt{0.75}$ & -1 & 0 \\
\hline & 0.5 & $\sqrt{0.75}$ & -0.5 & $-\sqrt{0.75}$ & & 0.5 & $-\sqrt{0.75}$ & -0.5 & $-\sqrt{0.75}$ \\
\hline & 0.5 & $\sqrt{0.75}$ & 0.5 & $-\sqrt{0.75}$ & & 0.5 & $-\sqrt{0.75}$ & 0.5 & $-\sqrt{0.75}$ \\
\hline & 0.5 & $\sqrt{0.75}$ & 0 & 0 & & 0.5 & $-\sqrt{0.75}$ & 0 & 0 \\
\hline \multirow[t]{7}{*}{3} & -0.5 & $\sqrt{0.75}$ & 1 & 0 & \multirow[t]{7}{*}{7} & 0 & 0 & 1 & 0 \\
\hline & -0.5 & $\sqrt{0.75}$ & 0.5 & $\sqrt{0.75}$ & & 0 & 0 & 0.5 & $\sqrt{0.75}$ \\
\hline & -0.5 & $\sqrt{0.75}$ & -0.5 & $\sqrt{0.75}$ & & 0 & 0 & -0.5 & $\sqrt{0.75}$ \\
\hline & -0.5 & $\sqrt{0.75}$ & -1 & 0 & & 0 & 0 & -1 & 0 \\
\hline & -0.5 & $\sqrt{0.75}$ & -0.5 & $-\sqrt{0.75}$ & & 0 & 0 & -0.5 & $-\sqrt{0.75}$ \\
\hline & -0.5 & $\sqrt{0.75}$ & 0.5 & $-\sqrt{0.75}$ & & 0 & 0 & 0.5 & $-\sqrt{0.75}$ \\
\hline & -0.5 & $\sqrt{0.75}$ & 0 & 0 & & 0 & 0 & 0 & 0 \\
\hline \multirow[t]{7}{*}{4} & -1 & 0 & 1 & 0 & & & & & \\
\hline & -1 & 0 & 0.5 & $\sqrt{0.75}$ & & & & & \\
\hline & -1 & 0 & -0.5 & $\sqrt{0.75}$ & & & & & \\
\hline & -1 & 0 & -1 & 0 & & & & & \\
\hline & -1 & 0 & -0.5 & $-\sqrt{0.75}$ & & & & & \\
\hline & -1 & 0 & 0.5 & $-\sqrt{0.75}$ & & & & & \\
\hline & -1 & 0 & 0 & 0 & & & & & \\
\hline
\end{tabular}


Table A.6: MWP Cuboidal Minimal Point Small Composite Design for D $(1,4)$ ( $z_{1}$ is a HTC factor, $x_{1}, x_{2}, x_{3}$, and $x_{4}$ are ETC factors)

\begin{tabular}{crrrrr} 
Whole-Plot & $z_{1}$ & $x_{1}$ & $x_{2}$ & $x_{3}$ & $x_{4}$ \\
\hline 1 & -1 & 1 & 1 & -1 & 1 \\
& -1 & -1 & 1 & 1 & 1 \\
& -1 & 1 & -1 & 1 & 1 \\
& -1 & 1 & 1 & 1 & -1 \\
& -1 & -1 & -1 & -1 & -1 \\
& -1 & 0 & 0 & 0 & 0 \\
& -1 & 0 & 0 & 0 & 0 \\
& -1 & 0 & 0 & 0 & 0 \\
& -1 & 0 & 0 & 0 & 0 \\
\hline 2 & 1 & 1 & -1 & 1 & -1 \\
& 1 & -1 & 1 & 1 & -1 \\
& 1 & 1 & 1 & -1 & -1 \\
& 1 & 1 & -1 & -1 & 1 \\
& 1 & -1 & -1 & 1 & 1 \\
& 1 & -1 & 1 & -1 & 1 \\
& 1 & 0 & 0 & 0 & 0 \\
& 1 & 0 & 0 & 0 & 0 \\
& 1 & 0 & 0 & 0 & 0 \\
\hline 3 & 0 & -1 & 0 & 0 & 0 \\
& 0 & 1 & 0 & 0 & 0 \\
& 0 & 0 & -1 & 0 & 0 \\
& 0 & 0 & 1 & 0 & 0 \\
& 0 & 0 & 0 & -1 & 0 \\
& 0 & 0 & 0 & 1 & 0 \\
& 0 & 0 & 0 & 0 & -1 \\
& 0 & 0 & 0 & 0 & 1 \\
& 0 & 0 & 0 & 0 & 0 \\
\hline & & & &
\end{tabular}


Table A.7: MWP Small Composite Design with Complete Plackett-Burman Factorial for $\mathrm{D}(1,4)$

$$
\text { ( } z_{1} \text { is a HTC factor, } x_{1}, x_{2}, x_{3} \text {, and } x_{4} \text { are ETC factors) }
$$

\begin{tabular}{crrrrr} 
Whole-Plot & $z_{1}$ & $x_{1}$ & $x_{2}$ & $x_{3}$ & $x_{4}$ \\
\hline 1 & -1 & -1 & -1 & 1 & -1 \\
& -1 & -1 & -1 & -1 & -1 \\
& -1 & -1 & 1 & -1 & 1 \\
& -1 & 1 & -1 & 1 & 1 \\
& -1 & 1 & 1 & 1 & -1 \\
& -1 & 1 & 1 & -1 & 1 \\
& -1 & 0 & 0 & 0 & 0 \\
& -1 & 0 & 0 & 0 & 0 \\
& -1 & 0 & 0 & 0 & 0 \\
\hline 2 & 1 & -1 & -1 & -1 & 1 \\
& 1 & -1 & 1 & 1 & 1 \\
& 1 & -1 & 1 & 1 & -1 \\
& 1 & 1 & -1 & 1 & 1 \\
& 1 & 1 & -1 & -1 & -1 \\
& 1 & 1 & 1 & -1 & -1 \\
& 1 & 0 & 0 & 0 & 0 \\
& 1 & 0 & 0 & 0 & 0 \\
& 1 & 0 & 0 & 0 & 0 \\
\hline 3 & 0 & -1 & 0 & 0 & 0 \\
& 0 & 1 & 0 & 0 & 0 \\
& 0 & 0 & -1 & 0 & 0 \\
& 0 & 0 & 1 & 0 & 0 \\
& 0 & 0 & 0 & -1 & 0 \\
& 0 & 0 & 0 & 1 & 0 \\
& 0 & 0 & 0 & 0 & -1 \\
& 0 & 0 & 0 & 0 & 1 \\
& 0 & 0 & 0 & 0 & 0 \\
\hline & & & & &
\end{tabular}


Table A.8: MWP Cuboidal Central Composite Design for $\mathrm{D}(1,4)$ ( $z_{1}$ is a HTC factor, $x_{1}, x_{2}, x_{3}$, and $x_{4}$ are ETC factors)

\begin{tabular}{crrrrr} 
Whole-Plot & $z_{1}$ & $x_{1}$ & $x_{2}$ & $x_{3}$ & $x_{4}$ \\
\hline 1 & -1 & -1 & -1 & -1 & 1 \\
& -1 & 1 & -1 & -1 & -1 \\
& -1 & -1 & 1 & -1 & -1 \\
& -1 & 1 & 1 & -1 & 1 \\
& -1 & -1 & -1 & 1 & -1 \\
& -1 & 1 & -1 & 1 & 1 \\
& -1 & -1 & 1 & 1 & 1 \\
& -1 & 1 & 1 & 1 & -1 \\
& -1 & 0 & 0 & 0 & 0 \\
\hline 2 & 1 & -1 & -1 & -1 & -1 \\
& 1 & 1 & -1 & -1 & 1 \\
& 1 & -1 & 1 & -1 & 1 \\
& 1 & 1 & 1 & -1 & -1 \\
& 1 & -1 & -1 & 1 & 1 \\
& 1 & 1 & -1 & 1 & -1 \\
& 1 & -1 & 1 & 1 & -1 \\
& 1 & 1 & 1 & 1 & 1 \\
& 1 & 0 & 0 & 0 & 0 \\
\hline 3 & 0 & -1 & 0 & 0 & 0 \\
& 0 & 1 & 0 & 0 & 0 \\
& 0 & 0 & -1 & 0 & 0 \\
& 0 & 0 & 1 & 0 & 0 \\
& 0 & 0 & 0 & -1 & 0 \\
& 0 & 0 & 0 & 1 & 0 \\
& 0 & 0 & 0 & 0 & -1 \\
& 0 & 0 & 0 & 0 & 1 \\
& 0 & 0 & 0 & 0 & 0 \\
\hline & & & &
\end{tabular}




\section{B Summary of Box-Behnken Designs}

Table B.1: Summary of Box-Behnken Design Size by Construction Method.

( $w$ is the number of HTC factors, $k$ is the number of ETC factors, $m$ is the number of whole-plots, and $n$ is the number of subplot runs per whole-plot)

\begin{tabular}{|c|c|c|c|c|c|}
\hline \multicolumn{2}{|c|}{ Factors } & \multicolumn{2}{|c|}{ VKM } & \multicolumn{2}{|c|}{ MWP } \\
\hline$w$ & $k$ & $m$ & $n$ & $m$ & $n$ \\
\hline 1 & 1 & & & & \\
\hline 1 & 2 & 4 & 4 & 3 & 5 \\
\hline 1 & 3 & 4 & 12 & 3 & 13 \\
\hline 1 & 4 & 4 & 24 & 3 & 25 \\
\hline 2 & 1 & 9 & 2 & 9 & $\overline{2}$ \\
\hline 2 & 2 & 10 & 4 & 9 & 5 \\
\hline 2 & 3 & 10 & 12 & & \\
\hline 2 & 4 & 10 & 8 & & \\
\hline 3 & 1 & 19 & 2 & 19 & 2 \\
\hline 3 & 2 & 20 & 4 & 19 & 5 \\
\hline 3 & 3 & 19 & 4 & & \\
\hline 3 & 4 & 21 & 4 & & \\
\hline
\end{tabular}

